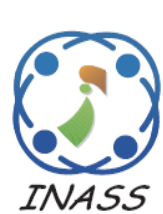

\title{
Aerial Image Enhancement based on YCbCr Color Space
}

\author{
Hana H. kareem $^{1 *}$ \\ Rana T. Saihood ${ }^{1}$ \\ ${ }^{I}$ Department of Physics, College of Education, Mustansiriyah University, Baghdad, Iraq \\ * Corresponding author's Email: hanakr2007@uomustansiriyah.edu.iq
}

\begin{abstract}
Enhancing aerial images plays an important role in many remote sensing applications; aerial images are often affected by dust, smoke, and fog. In this study, a new method was proposed to enhance aerial images. The proposed method is enhanced the light value (Y) in the $\mathrm{YCbCr}$ color transform by using the Sigmoid function mapping and Adaptive Histogram Equalization techniques, While the chromatic compounds (CbCr) are enhanced by Dark Channel Prior (DCP). To know the efficiency of enhancement, the no-reference quality measures as Entropy and Average Gradient (AG) have been calculated, which are used to see the quality of the proposed method in comparison with Image Entropy and Information Fidelity (IEIF), Multiscale Retinex with color restoration (MSRCR), Color Enhancement Scene Estimation (CESE), traditional (DCP), and Histogram Equalization (HE). From the results, it is found that the proposed method has succeeded in enhancing the aerial images better than other methods. Where it had high values for the rate for Entropy (7.73) and AG (68.75).
\end{abstract}

Keywords: Aerial image enhancement, Dark channel prior, $\mathrm{YCbCr}$ color space, Retinex algorithm.

\section{Introduction}

Enhance and restore distorted color images an important area of digital image processing [1-3]. This area includes noise, distortion in lighting, and lack of contrast. The atmosphere interacts with light due to the formation of the atmosphere under stable conditions, air contains many tiny particles called aerosols. Most are 0.1 micrometers in diameter. So they are invisible, except when clustered in huge quantities. One of the common obstacles is the aerial images that are degraded by the mote. mote is suspended quite fine solid or liquid particles. In the atmosphere with very low fall velocity, it is mostly between $(102-100) \mu m$ in size. Furthermore, rain, fog, dust, spray, snow, and smoke are examples of aerosols [4]. It has an important role in many applications of aerial image in aircraft, identifying and distinguishing targets and other fields.

Several studies have included improving aerial and hazy images. One of the most traditional methods of improving lighting and contrast is histogram equalization [5], this technique is adjusting the intensity of an image to improve contrast with this modification, the intensity can be best distributed on the image. This allows regions with low local contrast to having good contrast.

In [6] they suggested single enhanced for hazy images. This method depending on applying a white balance, a contrast-enhancing procedure and minimize artifacts by using a multistate fashion, depending on Laplacian pyramid representation, in this method improved the video clips distorted by haze. Main of the basic and important methods that are considered uncomplicated algorithms are prior dark channel prior, It automatically improves the high haze areas, and the haze-free areas are not distorted [7].

Some algorithms depending on non-local prior [8], this method assumes the color data of a haze free image are a good approximation depending on a few hundred singular data, which consist of strict clusters in the basic RGB color model.

It can be considered that the pixels in a certain group are often nonlocal, that is spread over the entire plane of the image and which are existing at 
different distances from the camera. Each color cluster in the free haze image becomes a line in RGB space, This form can get good improvement.

Some hazy image enhancement is entropy dependent [9], in this method the atmospheric light is represented by quadtree subdivision and the transmission is dependent on an objective function that is comprised of information quality by using weighted least squares optimization.

A common way to enhance brightness and contrast is an algorithm multi-scale retinex algorithm with color restoration [10], it can be used to improve the hazy image, this algorithm relies on the high-pass filter (Gaussian filter) With the Logarithm mapping. But image enhancement often suffers from Hula effected. However, it can be characterized and flown to improve underwater and hazy images. Yong $\mathrm{Xu}$, et al. [11] introduced an algorithm to improve distorted images captured under fog and hazy weather, which affects the tracking, detection, and recognition of targets they introduced the existing image de-fogging algorithms, including image recovery algorithms fusion-based defogging algorithms, and image contrast enhancement algorithms. To enhance edge efficiency and preservation various transmission enhancement methods have been suggested. The results illustrate the comparison of several typical enhanced dark channel prior based defogging algorithms. The bilateral filtering, soft matting method, and guided image filtering method acquired close results, depending on guided image filtering that gets a good enhancement.

Jin-Bao Wang, et al. [12] introduced a single image dehaze algorithm that is used a dark channel prior and a physical model. The selection light value in atmospheric by direct dependence on the color information and contrast in the color image. They studied atmospheric light rely on the variable, that quietly weakens regions in the color image which do not correspond to the previously dark channel. Furthermore, they suggested a good transmission estimation method to reduce the processing time. Taking into consideration a subjective assessment, the image quality was also evaluated using three scales PSNR, MSE, and average gradient. Their experiments showed that their approach had satisfactory results in improving aerial images. In many studies, the color correction of compounds has been used as an important basis for enhancement [13], For aerial image enhancement by using Color Enhancement and Scene Estimation (CESE) [14], Fayaz Ali, et al. Suggested algorithm includes Enhancement color contrast and color correction depending on Piecewise Linear Transformation
(PWLT). All treatments were performed on the RGB compounds individually without the use of color transformation. In their results, a good contrast improvement was obtained in the aerial images. In aerial images, distortion in lighting and average distortion in colors may occur. In all most previous methods, enhancement using chromatic compounds may cause color distortion due to the high correlation of RGB components. One of the strengths of the Suggested method is that it tries to improve the lightness with the sigmoid function separately for the lightness component, which represents $80 \%$ of the bytes with consistent improvement of colors. Most of the previous techniques do not improve lighting independently, so areas remain dark or highly lit, that happens to the color distortion.

\section{Proposed method}

An algorithm has been proposed to improve aerial images based on $\mathrm{YCbCr}$-space, where three techniques were used, which are DCP combined with sigmoid mapping and adaptive histogram equalization and as follows:

\subsection{Applied DCP on CbCr component}

The proposed method is based on the improvement of aerial images using $\mathrm{YCbCr}$ color space, Where DCP technology is used on $\mathrm{CbCr}$ chromatic compounds.

This is done by applying the DCP algorithm to the color image, then converting the image to $\mathrm{YCbCr}$ space, and extracting the improved $(\mathrm{CbCr})$. Compounds.

The general model describing dust or fog in a scene is given in relation $[7,15]$ :

$$
I(x)=J(x) \operatorname{tr}(x)+A c(1-\operatorname{tr}(x))
$$

Where $I$ is the intensity of the hazy image, $J$ is the radiance in the scene (the true color that wants to A being atmospheric light, and tr is the transmission. One of the most common algorithms for removing blur from images is the DCP, which is based on the assumption Most of the non-sky patches are for the free haze outdoor images, which have very low intensities in at least one of the RGB components.

Thus, DCP for an arbitrary image $J i$ is defined as [7]:

$$
J_{\text {dark }}(x)=\min _{i \epsilon\{r, g, b\}}\left(\min _{y \epsilon \Omega(x)}(J i(y))(2)\right.
$$

Where $J i$ is a color image of $\mathrm{J}$ and $\Omega(x)$ being a 
local patch has $\mathrm{x}$ center Depending on the DCP algorithm, the intensity of the dark channel of Ji is low and go to be zero where Ji is an outdoor hazefree image, except the bright region:

$$
J_{\text {dark }}(x) \cong 0
$$

Thus the transmission value can be determined by [7]:

$$
\operatorname{tr}(x)=1-w_{\min y \epsilon} \Omega(x)\left(\min _{i \in\{r, g, b\}} \frac{I^{c}(y)}{A}\right)(4)
$$

The transmission can be refined by using map soft mapping. If the haze is removed, the image appears unnatural, so the value $(0<w<1), w$ is fixed at 0.95 , and the best value of atmospheric light $A=0.1$, with patch size $(15 \times 15)$ [7]. Then recover the scene radiance by using [7]:

$$
J(x)=\frac{I(x)-A}{\max (\operatorname{tr}(x), 0.1)}+A
$$

One of the color spaces that depend on the human vision system Is $\mathrm{YCbCr}$ color space, the image enhanced can be converted using DCP algorithm From basic $\mathrm{R}_{p}, G_{p}, P_{p}$ space to $\mathrm{YCbCr}$ space, after that we keep only the color compounds hue and saturation $\left(\mathrm{R}_{p}\right.$ and $\left.\mathrm{Cr}_{p}\right)$. By using transform [16]:

$$
\begin{gathered}
\mathrm{Y}_{p}=0.298 \mathrm{R}_{p}+0.587 \mathrm{G}_{p}+0.114 \mathrm{~B}_{p} \\
\mathrm{Cb}_{p}=-0.147 \mathrm{R}_{p}-0.2880 \mathrm{G}_{p}+0.436 \mathrm{~B}_{p} \\
\mathrm{Cr}_{p}=0.6149 \mathrm{R}_{p}+0.514 \mathrm{G}_{p}-0.100 \mathrm{~B}_{p}
\end{gathered}
$$

\subsection{Applied Sigmod mapping and AHE on Y component}

The lightness component according to $\mathrm{YCbCr}$ is given by [16]:

$$
G=0.298 R+0.587 G+0.114 B
$$

After this, the lighting vehicle is converted using the sigmoid function according to [17]:

$$
G \mathrm{t}=1 /\left(1+\sqrt{\left.\frac{1-G}{G}\right)}\right.
$$

This conversion of the illumination composite increases the contrast of all areas of irregular lighting in the aerial photos, in a convenient manner. To validate the issues in continuous lighting and exposure, the idea relies on the compromise of a histogram and its contrast. The chance of re-charting the scenery histogram to a histogram that has a

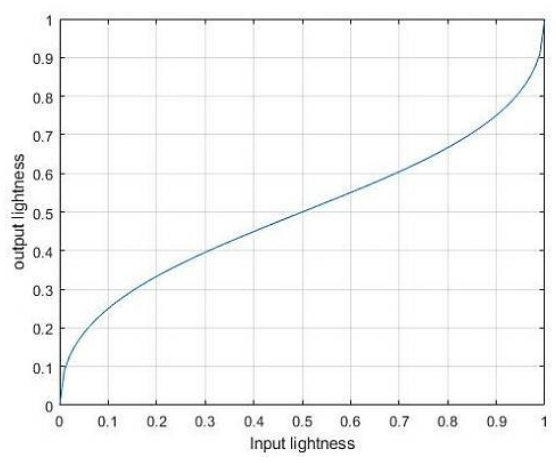

Figure. 1 The relationship between light input and output value according to Sigmoid function [17]

regular quantile work that is adjacent, and this is done through the use of AHE (Adaptive Histogram Equalization) [18] technique, AHE promotes tight image blocks and the contrast between each block is improved such that the processed histogram area coincides with a histogram that may be Binomial, Gaussian, Poisson or Rayleigh in the distribution provided. When we apply AHE technique to a $G$ function we get $G t_{e}$.

\subsection{The combination of enhancement compounds}

After the chromatic compounds $(\mathrm{Cb}$ and $\mathrm{Cr})$ are enhancing using DCP And improve the lighting (Gt) by using adaptive histogram equalization by sigmoid function All three compounds are combined Then reverse transform from $\mathrm{YCbCr}$ to $\mathrm{RGB}$. The transform is given by the equation [16]:

$$
\begin{gathered}
R e=G t_{e}+1.401 c r_{p} \\
G e=G t_{e}-0.394 c b_{p}-0.580 c r_{p} \\
B e=G t_{e} 2.032 c b_{p}
\end{gathered}
$$

Fig. 2 shows the block diagram of the proposed method for improving aerial images. And Fig. 3 illustrated these stages with an aerial image.

\section{Determine the quality assessment}

In this study, non-reference quality metrics were used to measure the quality of dust-distorted images. The first measure is the entropy, which increases the contrast in an image as given by [5]:

$$
\text { Entropy }=-\sum_{\mathrm{v}=0}^{255} \mathrm{P}(\mathrm{G}) \log \mathrm{P}(\mathrm{G})
$$

Where $G$ the total gray is level number and $P(G)$ is that the probability density function at intensity. 
The second measure is the average gradient, which measures the quality of the edge areas [19]:

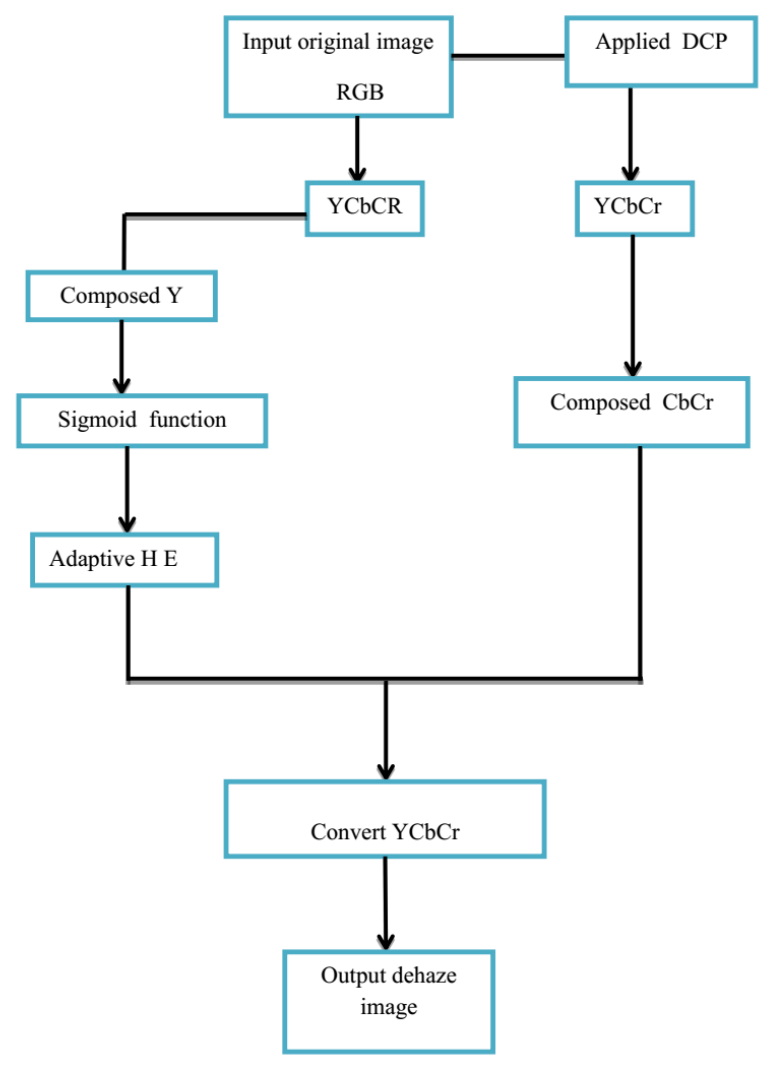

Figure. 2 Block diagram of the proposed method

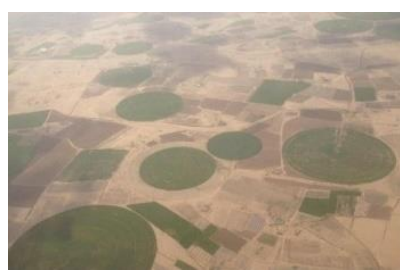

(a)

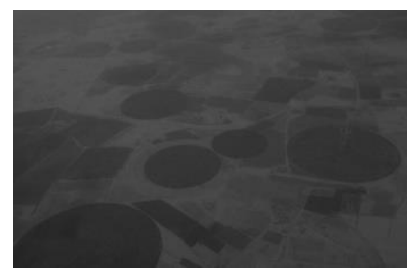

(b)

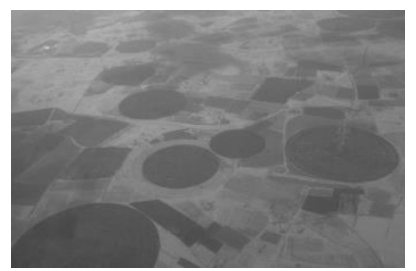

(c)

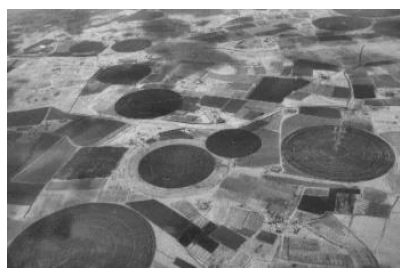

(d)

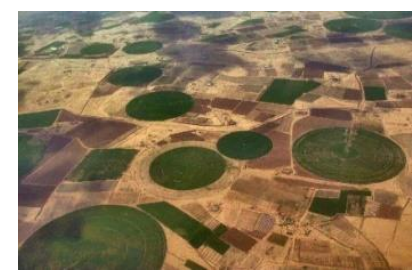

(e)

Figure. 3 (a) Original image (b) Lighting component $\mathrm{Y}$ according to $\mathrm{YCbCr}$ color space (c) enhancing the $\mathrm{Y}$ by relying on the Sigmoid function (d) enhancement by AHE (e)The final image of the enhancement after merging the lighting component in AHE image with the color components resulting from improving the original image using DCP

$$
\mathrm{AG}=\frac{1}{(u-1)(v-1)} \sum_{i=1}^{u-1} \sum_{j=1}^{v-1} \sqrt{\frac{\nabla_{i}^{2} G(i, j)+\nabla_{j}^{2} G(i, j)}{2}}
$$

Where $u, v$ is image size.

\section{Result and discussion}

In this study, the aerial images were improved by relying on the YCbCr color space, The proposed method has been compared with several other methods are MSRCR [10], HE [5], DCP [7], Image Entropy, and Information Fidelity (IEIF) [9], and CESE [14]. Four aerial images of type JPG and size $(960 \times 1280)$ were selected, captured from an airspace plane in Iraq were taken from an altitude of less than $5 \mathrm{~km}$ as shown in Fig. 4, Matlab R2020a 
has been used to perform all enhancement methods. The Fig. 6 to 9 show aerial images enhanced with proposed, MSRCR, HE, DCP, IEIF, and CESE depending on a subjective evaluation, we notice that the best way to enhance is due to the proposed and DCP algorithms. Table 1 shows the values of the unreferenced measures of average gradient and entropy, for the average values for average gradient the four images have been reached $(68.75,62.38$, $67.82,56.11,54.17$, and 59.28) respectively where was the highest value (68.75) for the proposed method followed by HE, MSRCR, CESE, DCP, and IEIF) respectively. This indicates the success of the proposed method in improving the edge regions in aerial images. In Table 1 , for the mean entropy values $(7.73,7.38,5.98,7.46,7.57$, and 7.58), and we note that the proposed method has the highest

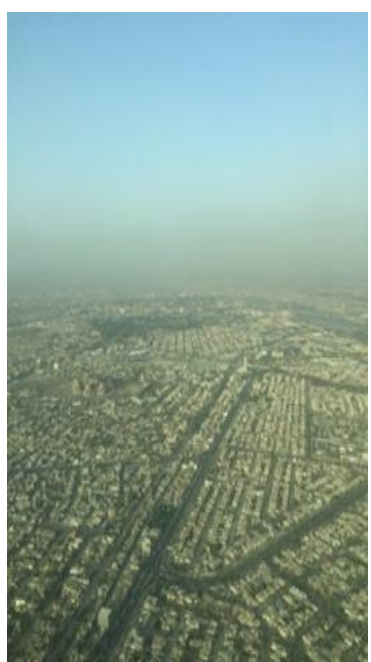

(1)

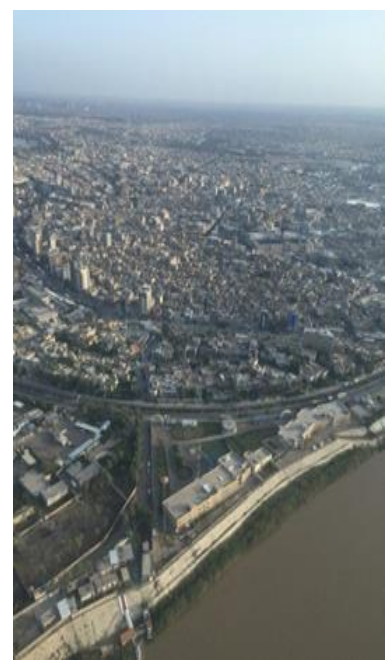

(2)

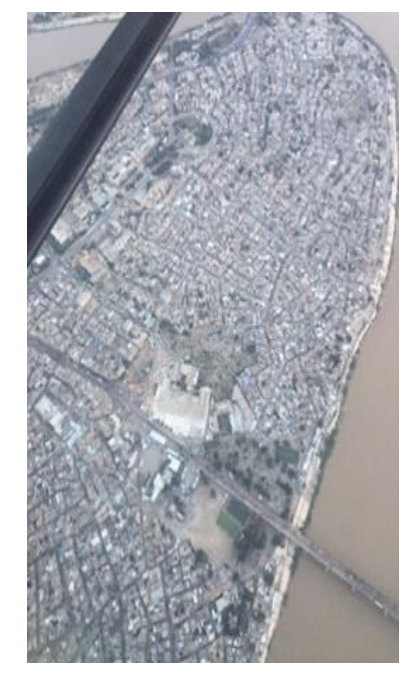

(3)

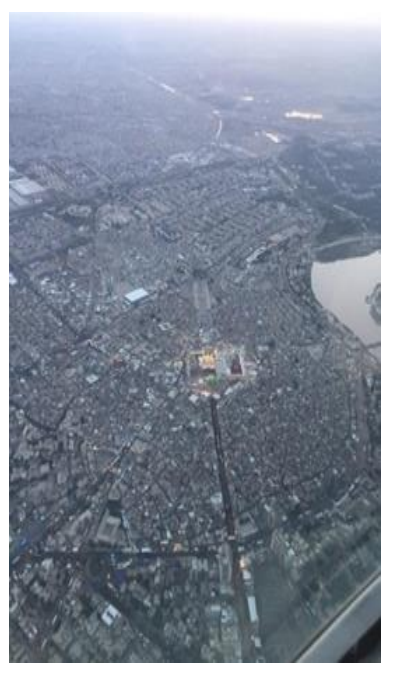

(4)

Figure. 4 Aerial images used in this study

Table 1 . The quality for all enhanced aerial images

\begin{tabular}{|c|c|c|c|c|c|}
\hline \multirow{2}{*}{ Methods } & \multicolumn{5}{|c|}{$\mathbf{A G}$} \\
\hline & İmage 1 & İmage 2 & İmage 3 & İmage 4 & Averege \\
\hline Sug. & 63.21 & 62.30 & 88.19 & 61.28 & 68.75 \\
\hline MSRCR & 58.31 & 68.85 & 61.76 & 60.60 & 62.38 \\
\hline HE & 59.68 & 58.53 & 90.90 & 62.16 & 67.82 \\
\hline DCP & 49.04 & 49.86 & 80.95 & 44.57 & 56.11 \\
\hline IEIF & 49.97 & 48.41 & 70.85 & 47.46 & 54.17 \\
\hline CESE & 51.07 & 54.46 & 76.34 & 55.26 & 59.28 \\
\hline \multirow{2}{*}{ Methods } & \multicolumn{5}{|c|}{ Entropy } \\
\hline & İmage 1 & İmage 2 & İmage 3 & İmage 4 & Averege \\
\hline Sug. & 7.69 & 7.71 & 7.89 & 7.64 & 7.73 \\
\hline MSRCR & 7.00 & 7.65 & 7.41 & 7.47 & 7.38 \\
\hline HE & 5.98 & 5.99 & 5.98 & 5.99 & 5.98 \\
\hline DCP & 7.91 & 7.45 & 7.25 & 7.25 & 7.46 \\
\hline IEIF & 7.70 & 7.50 & 7.75 & 7.31 & 7.57 \\
\hline CESE & 7.55 & 7.56 & 7.70 & 7.46 & 7.58 \\
\hline
\end{tabular}




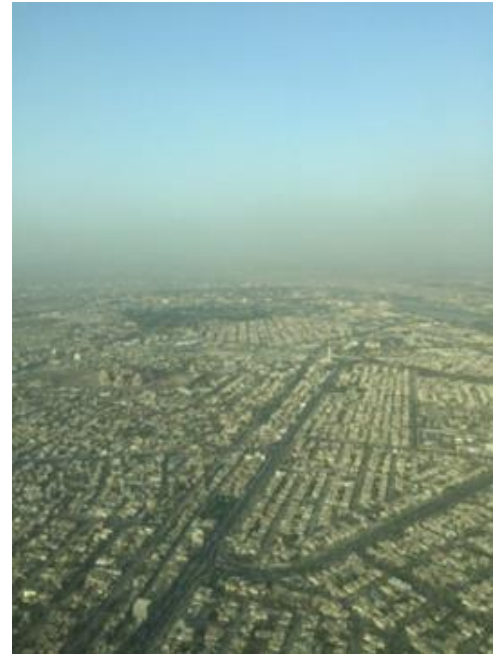

(a)

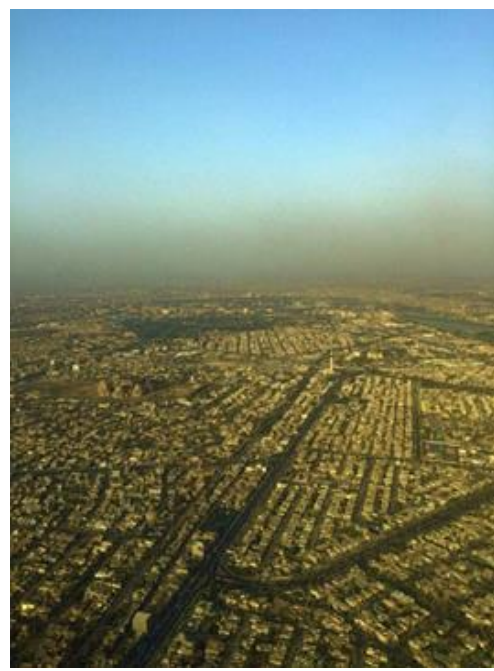

(d)

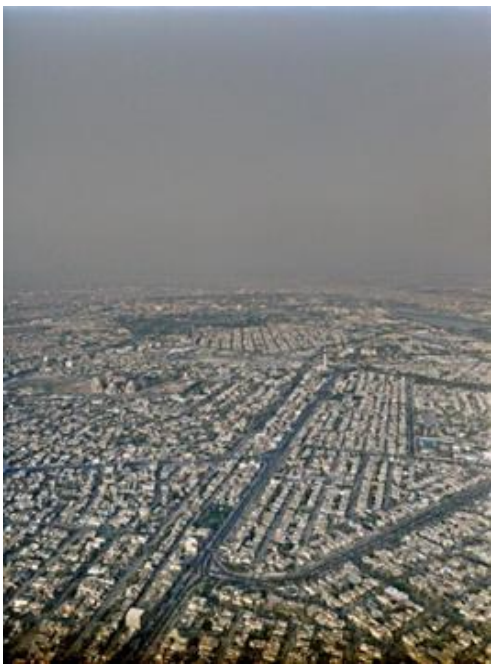

(b)

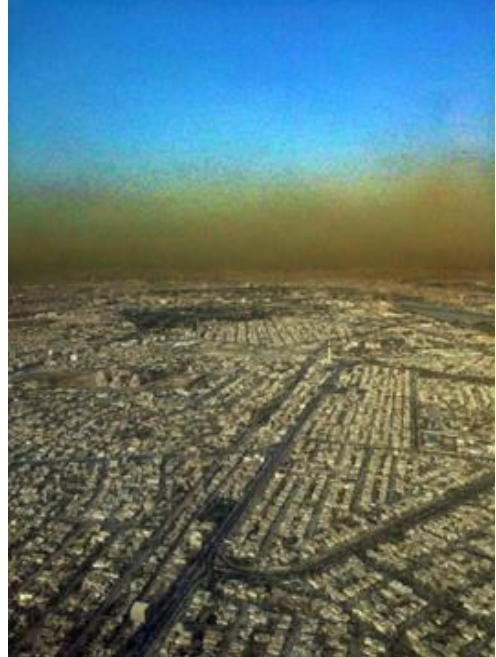

(e)

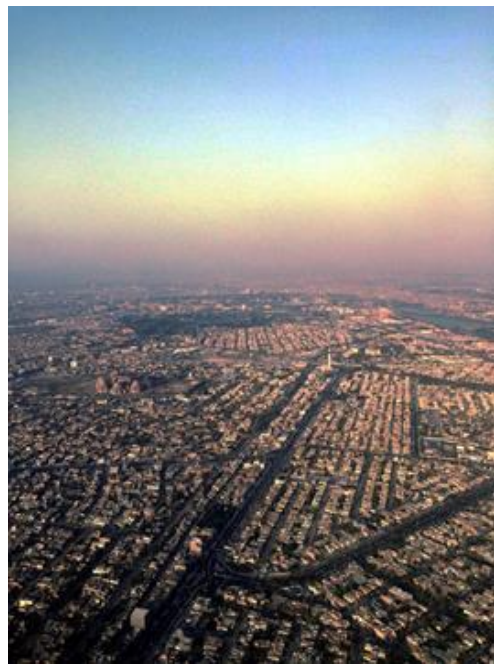

(c)

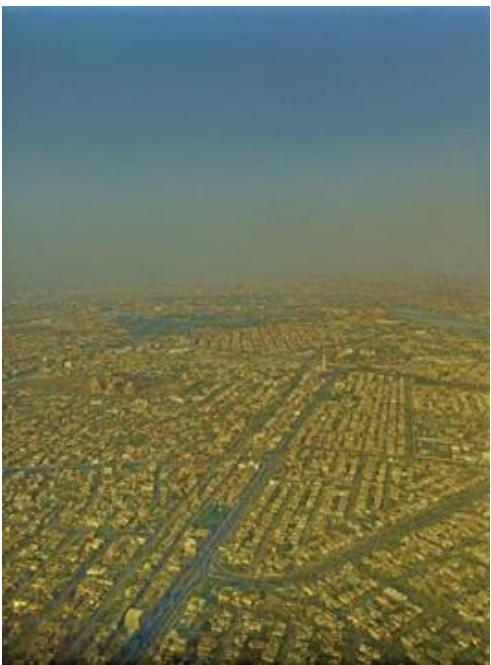

(f)

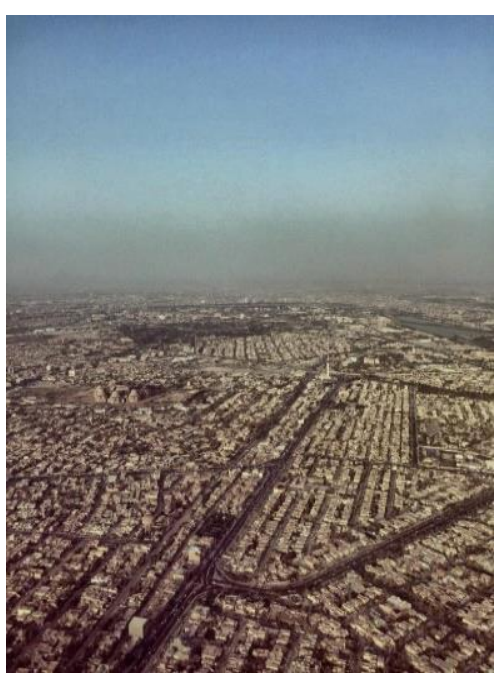

(g)

Figure. 6 First image in: (a) original image, and in ((b), (c), (d), (e), (f), and (g)) that are enhancement by different algorithms (MSRCR, HE, DCP, IEIF, proposed and CESE) respectively 


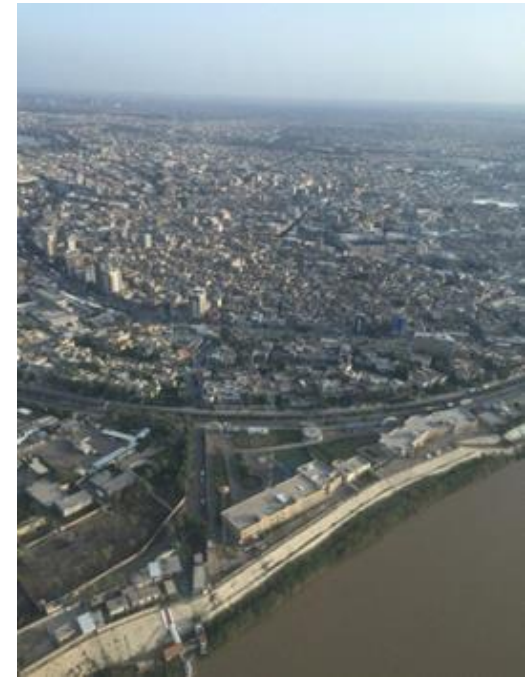

(a)

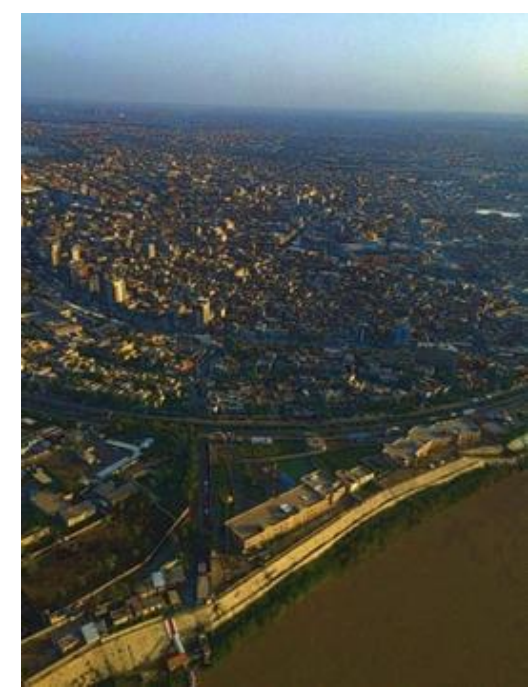

(d)

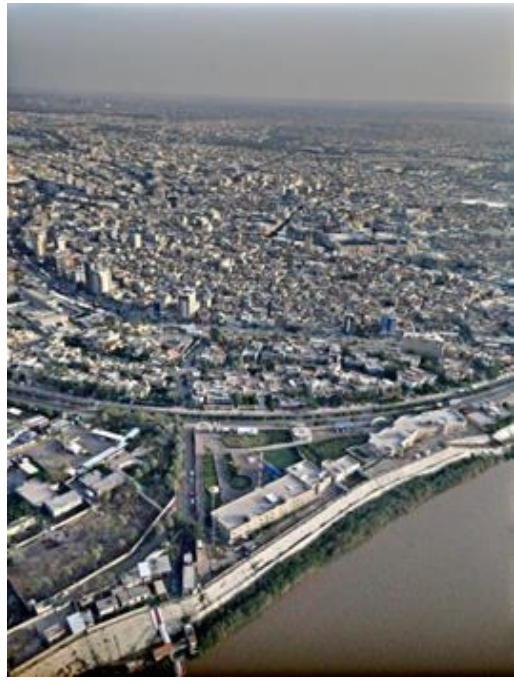

(b)

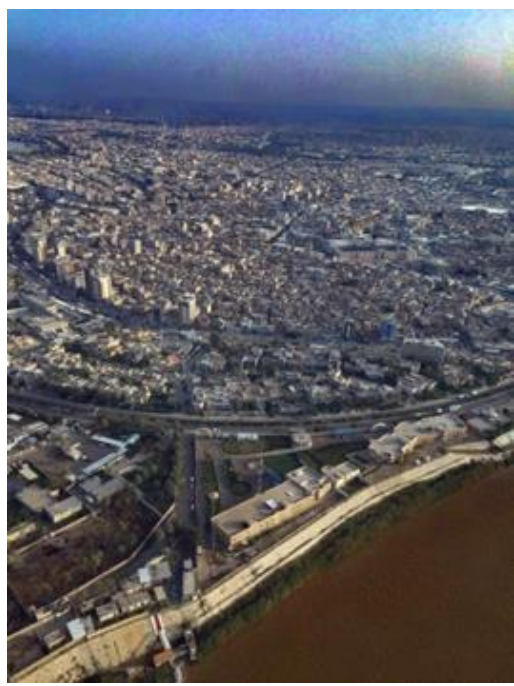

(e)

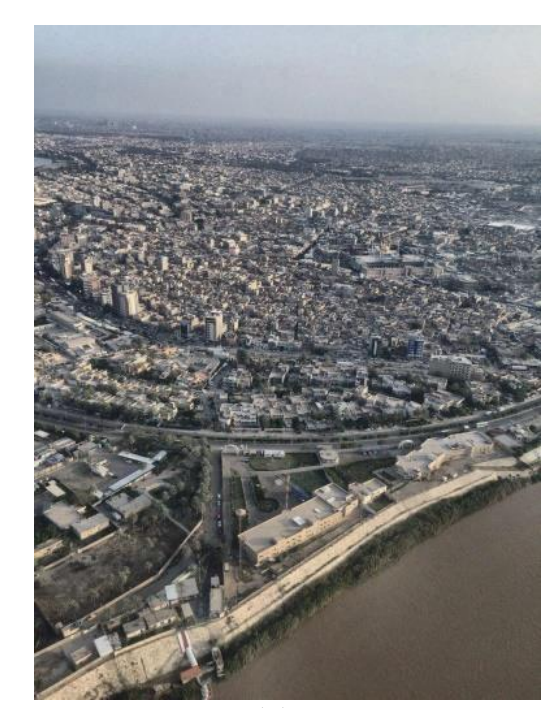

(g)

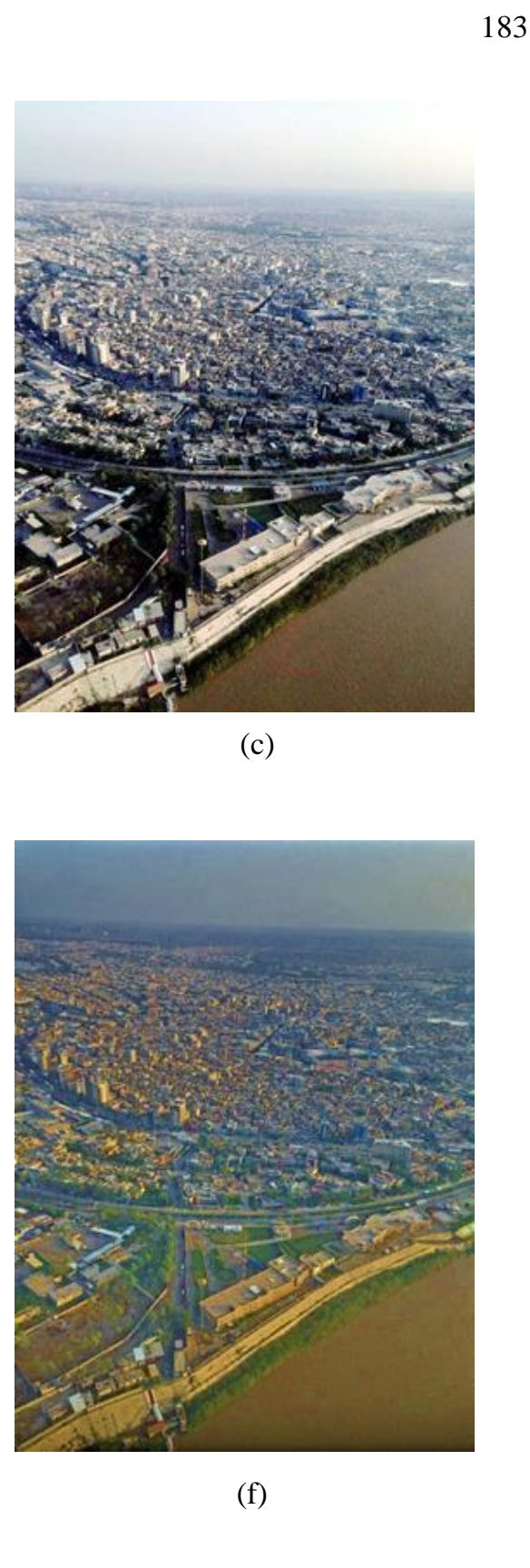

(c)

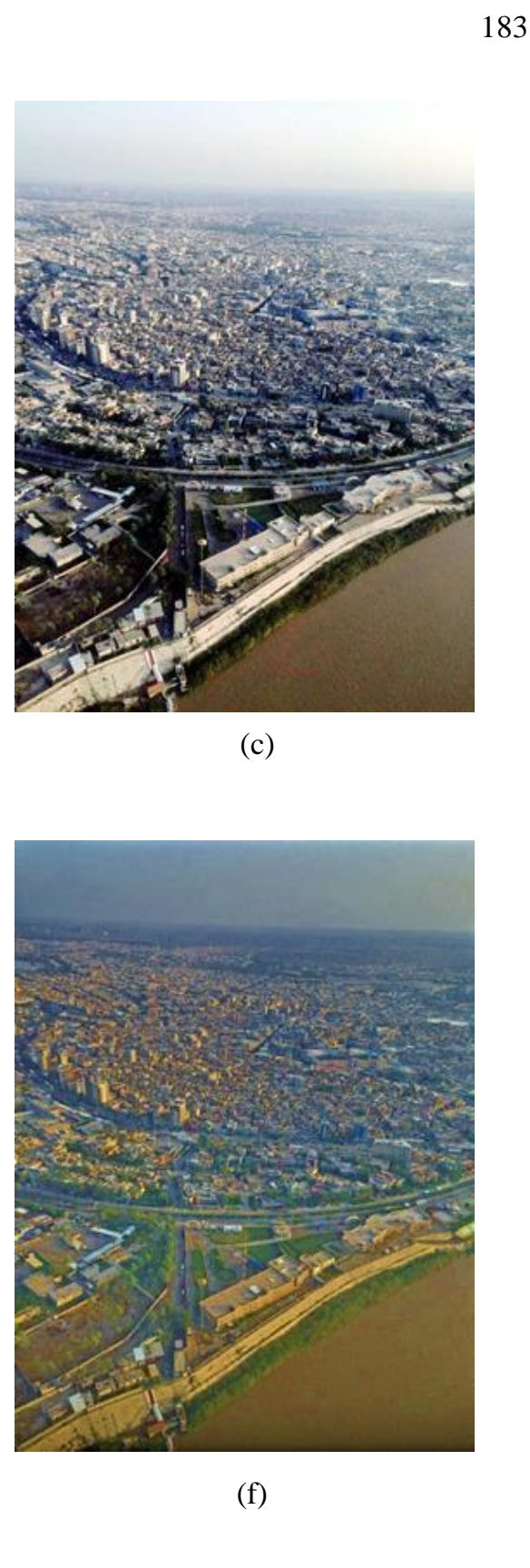

(f)

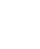




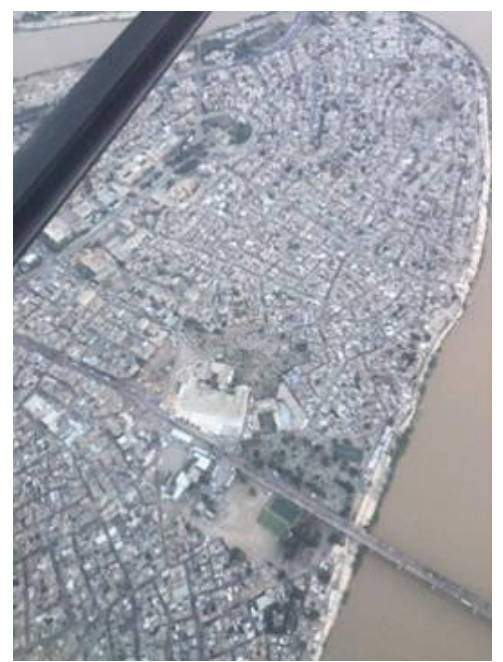

(a)

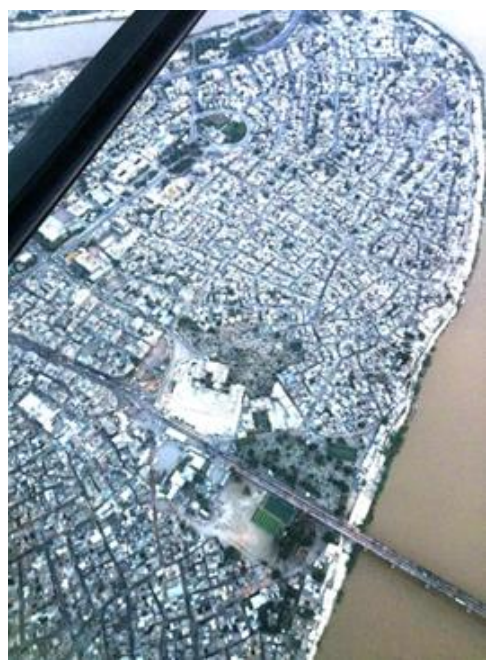

(d)

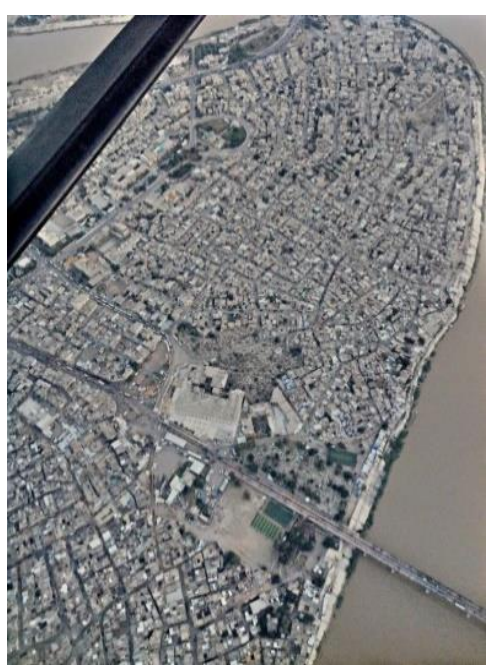

(b)

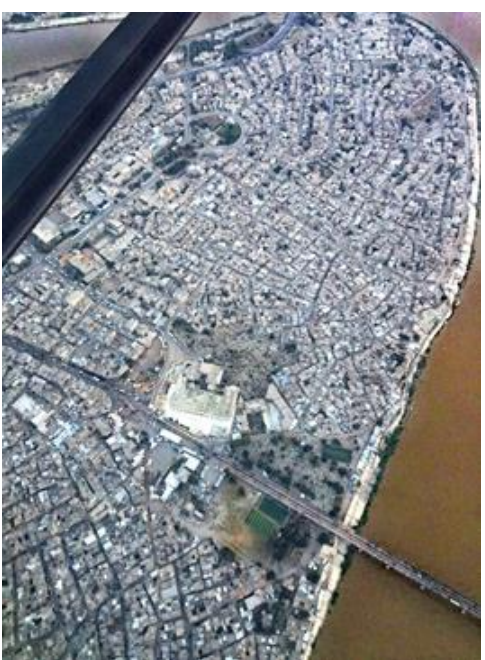

(e)

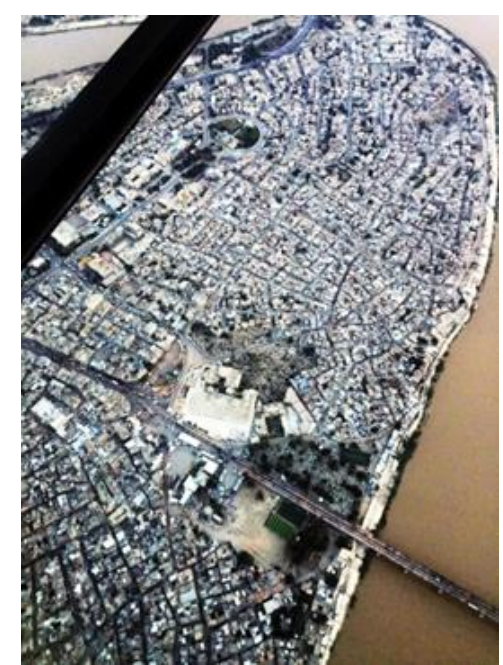

(c)

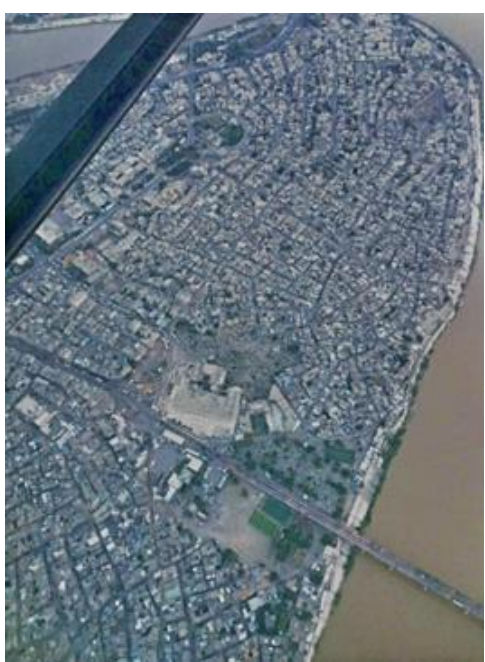

(f)

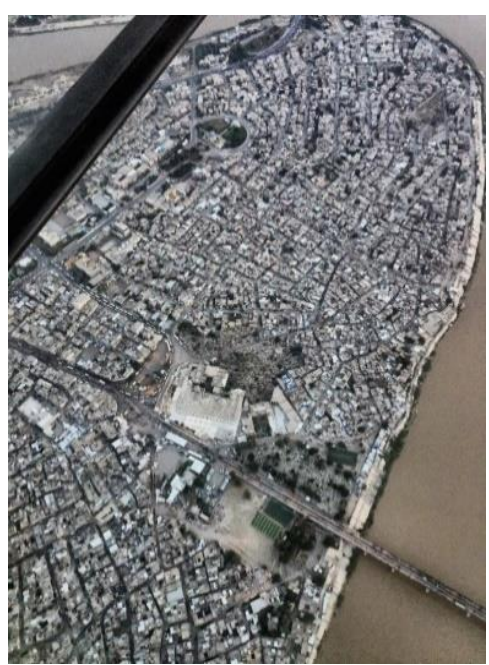

(g)

Figure. 8 Third image in: (a) original image, and in ((b), (c), (d), (e), (f), and (g)) that are enhancement by different algorithms (MSRCR, HE, DCP, IEIF, proposed and CESE) respectively 


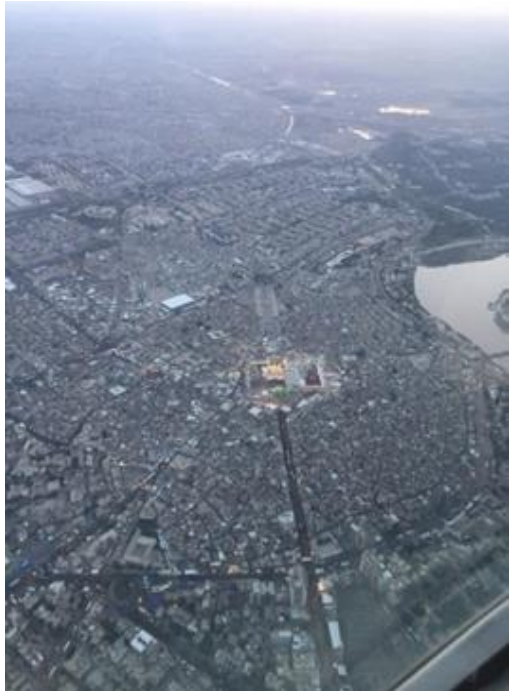

(a)

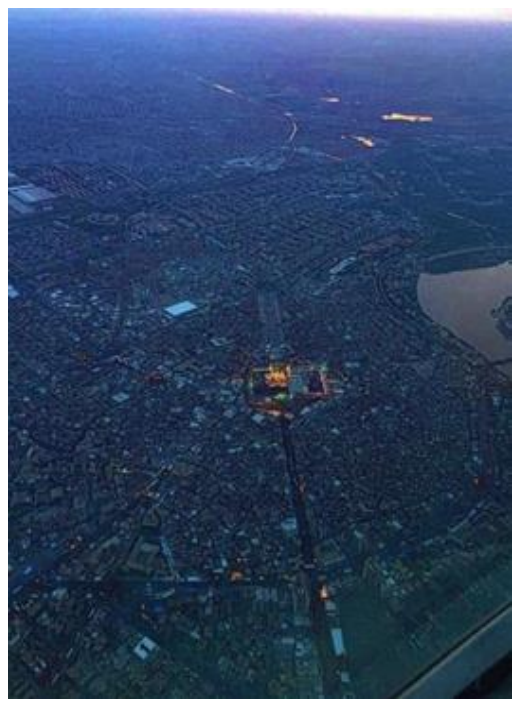

(d)

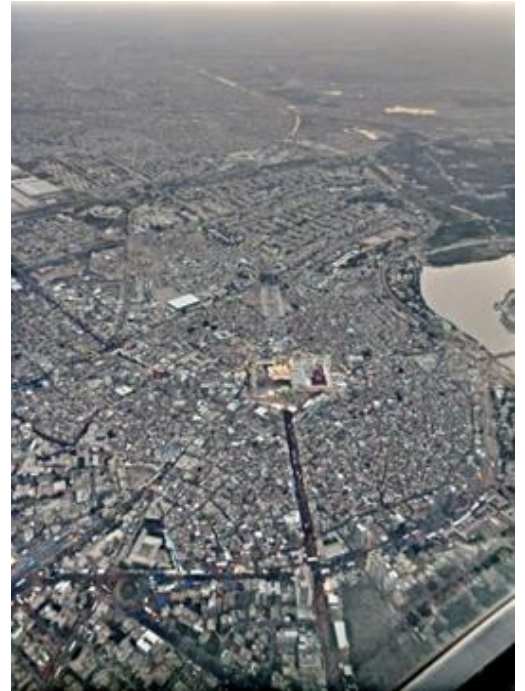

(b)

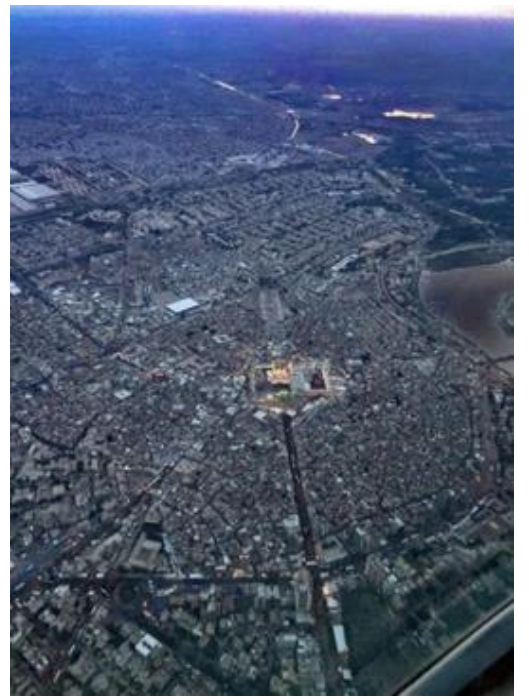

(e)

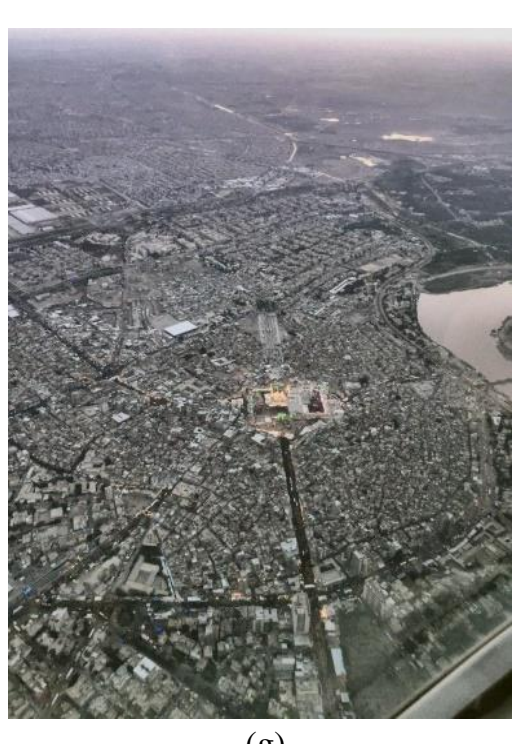

(g)

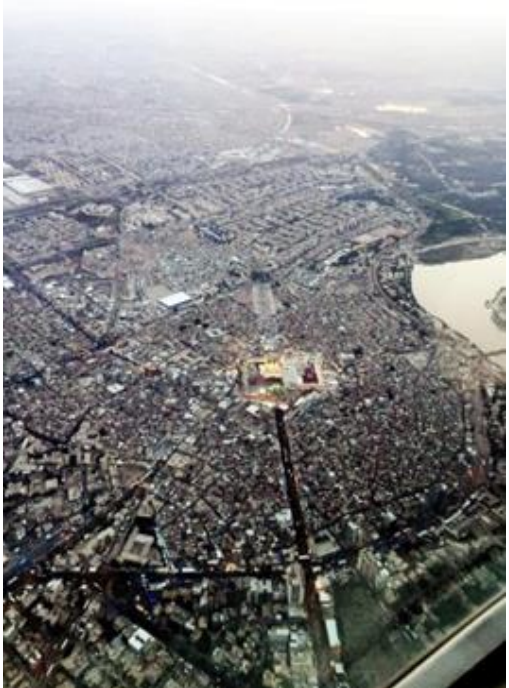

(c)

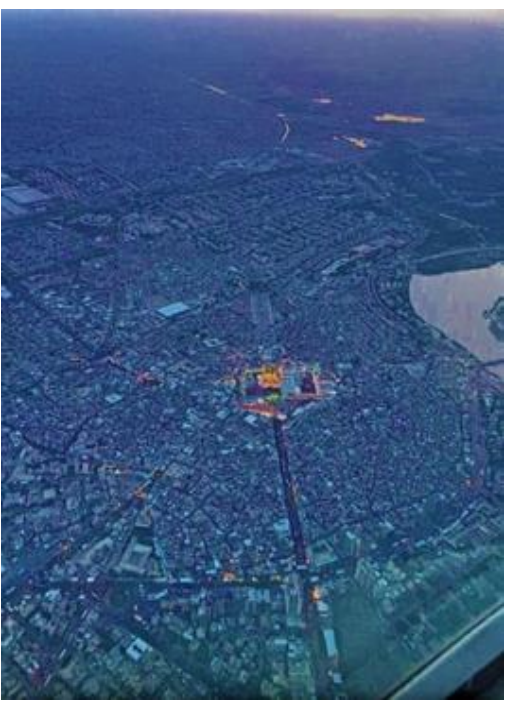

(f) 


\section{Conflicts of Interest}

The authors declare no conflict of interest.

\section{Author Contributions}

Hana H. Kareem has contributed to the design and implementation of the research by using Matlab. Rana T. Saihood had supervised the written paper and providing the necessary data. All authors approved the final version.

\section{References}

[1] Z. Ameer, H. Daway, and H. Kareem, "Enhancement underwater image using histogram equalization based on color restoration", Journal of Engineering and Applied Sciences, Vol. 14, No. 2, pp. 641-647, 2019.

[2] N. M. Mirza, H. H. Kareem, and H. G. daway, "Low lightness enhancement using nonlinear filter based on power function", Journal of Theoretical and Applied Information Technology, Vol. 96. No. 1, pp. 61-70, 2019

[3] K. Ghada, Z. Abood, H. Kareem, and H. Dowy, "Blurred Image Restoration with Unknown Point Spread Function", Al-Mustansiriyah Journal of Science, Vol. 29, No. 1, 2019.

[4] H. Daway, F. Mohammed, and D. Abdulabbas, "Aerial image enhancement using modified fast visibility restoration based on sigmoid function", Advances in Natural and Applied Sciences, Vol. 10, No. 11, pp. 16-22, 2016.

[5] C. Rafael and E. Richard, "Digital Image Processing", Prentice-Hall, 2002.

[6] C. Ancuti, "Single Image Dehazing by MultiScale Fusion", IEEE Transactions on Image Processing, Vol. 22, No. 8, 2013.

[7] K. He, J. Sun, and X. Tang, "Single Image Haze Removal Using Dark Channel Prior", IEEE Transactions on Pattern Analysis and Machine Intelligence, Vol. 33, No. 12, 2011.

[8] D. Berman and S. Avidan, "Non-Local Image Dehazing", IEEE Conference on Computer Vision and Pattern Recognition, 2016.

[9] Park, H. Park, D. K Han, and H. Ko, "Single Image Dehazing with Image Entropy and Information Fidelity", In: Proc. of IEEE International Conf. on Image Processing (ICIP), 2014.

[10] D. Jobson, Z. Rahman, and G. Woodell, "Properties and Performance of a Center / Surround Retinex", IEEE Transactions on Image Processing, Vol. 6, No. 3, 1997.
[11] Y. Xu, J. Wen, L. Fei, and Z. Zhang "Review of Video and Image Defogging Algorithms and Related Studies on Image Restoration and Enhancement", IEEE Access Journal, Vol. 4, 2016.

[12] J. Wang, N. He, L. Zhang, and K. Lu, "Single Image Dehazing With a Physical Model and Dark Channel Prior", Neurocomputing, Vol. 149, 2015.

[13] R. Habeeb, H. Kareem, and A. Ahmed, "Lightness enhancement for video images based on nonlinear filter", In: Proc. of IEEE First International Conf. of Computer and Applied Sciences (CAS), 2019.

[14] F. Adharejo, Zhou, F. Deeba, and Y. Du, "A Color Enhancement Scene Estimation Approach for Single Image Haze Removal," IEEE Geoscience and Remote Sensing Letters, Vol. 17, No. 9, 2020.

[15] R. Tan, "Visibility in bad weather from a single image", In: Proc. of 2008 IEEE Conference on Computer Vision and Pattern Recognition, 2008.

[16] S. Wine and R. Horne, "The Color Image Processing Hand Book", International Thomson, 1998.

[17] R. Habeeb and H. Kareem, "Video Images Enhanced by using Sigmoid-Logarithm Transform", In: Proc. of IOP Conf. Series: Materials Science and Engineering, 2020.

[18] S. Pizer, R. Johnston, J. Ericksen, B. Yankaskas, and K. Muller, "Contrast-limited adaptive histogram equalization: Speed and effectiveness", In: Proc. of 1st Conf. Vis. Biomed. Comput., 1990.

[19] J. Zhang, S. Ong, and M. Le, "Kurtosis-based no-reference quality assessment of JPEG2000 images", Signal Processing: Image Communication, Vol. 26, No. 1, 2011. 